\title{
PERFIL OROFACIAL DE LAS PERSONAS MAYORES INSTITUCIONALIZADAS DE LA CIUDAD DE BUCARAMANGA Y SU ÁREA METROPOLTTANA
}

${ }^{1}$ William Fernando Bermúdez, ${ }^{2}$ Sonia Constanza Concha Sánchez, ${ }^{3}$ Diana Marina Camargo Lemos.

${ }^{1}$ Odontólogo, U. Santo Tomás, ${ }^{2}$ Odontóloga, Especialista en Comunicación para la salud y el bienestar, Grupo SIBAM, Facultad de Odontología U. Santo Tomás, ${ }^{3}$ M.Sc. Epidemiología, Coordinadora Grupo SIBAM, Facultad de Odontología U. Santo Tomás, Prof. Auxiliar E. Fisioterapia, UIS

Autor responsable de correspondencia: Sonia Constanza Concha Sánchez

e-mail: Sococosa@ustabuca.edu.co

\section{RESUMEN}

Objetivo: El propósito del estudio fue establecer el perfil orofacial de las personas adultas mayores institucionalizados de la ciudad de Bucaramanga y su área metropolitana.

Materiales y Métodos: Se realizó un estudio observacional descriptivo en adultos mayores de 60 años, residentes permanentes de instituciones geriátricas, que aceptaron participar en el estudio. Se estableció la prevalencia de alteraciones en el examen facial, mucosa oral, articulación temporomandibular (ATM), índice COP(d), Indice de caries radicular (RCI), estado periodontal (CPITN), higiene oral y estado protésico.

Resultados:Se examinaron 284 adultos mayores, 55\% del género masculino con un promedio de edad de 77.7 años, carecían de seguridad social el 35\%; consumía algún medicamento el 65\% y el 39\% registró hipertensión arterial. Las mejillas presentaron la mayor frecuencia de anormalidad (80.6\%); en mucosa oral, la lengua presentó el mayor número de lesiones (66.2\%) y la alteración de ATM más frecuente fue el ruido articular (60\%).

El promedio de COP fue $26.4 \pm 3.3$, el Indice de Caries radicular (RCI) tuvo un promedio de 31 y una mediana de 40 , con un rango entre 0 y 50 . El estado periodontal de moderado a severo se presentó en el $63 \%$ y edentulismo total en $55 \%$.

Conclusión: El adulto mayor institucionalizado presenta una condición oral muy deficiente. Su manejo y control requiere de investigaciones multidisciplinarias que orienten las estrategias de intervención en una forma integral. [Bermúdez W, Concha S, Camargo D. Perfil orofacial de las personas mayores institucionalizadas de la ciudad de Bucaramanga y su área metropolitana. Ustasalud 2003;1:13-19]

PALABRAS CLAVES: Adulto mayor institucionalizado, odontogeriatría, condición oral

\section{Orofacial Profile In Institutionalized Elderly People In The City Of Bucaramanga And Metropolitan Area}

\begin{abstract}
Purpose: The purpose of the study was to establish the orofacial profile of institutionalized elderly people from Bucaramanga and metropolitan area.

Material and Methods: A descriptive study in institutionalized adults oldest than 60 years was carried out, who accepted participate. A whole orofacial profile were established by facial, mucosa and TMJ examination, DMFT index, RCI, CPITN, oral hygiene and prosthesis status.

Results: Two hundred and eigthy four elderly persons was evaluated, $55 \%$ male, with an average of total age 77.7 years old; $35 \%$ have no social security. The medical condition more frequent was hypertension (39\%) and nervous system disease (38\%); medical attendance (57.7\%) and pharmacologic treatment (65\%).

In a facial examination cheek, exhibit high prevalence of alterations (80\%), TMJ noises was (60\%). Medial pterigoid muscle exhibit pain in $44 \%$ and in the oral mucosa, white lesion on lateral borders of the tongue was the more frequent (66.2\%).

The average of DMFT index was $26.4 \pm 3.3$, RCI index 31, median 40 and range between 0 and 50 . Mild to severe periodontal condition by CPITN index was $63 \%$ and total edentulism was $55 \%$.

Conclusion: Institutionalized elderly people have a deficient oral condition. Its management and control request multidisciplinary research and integral intervention strategies.
\end{abstract}

KEY WORDS: Institutionalized elderly people, odontogeriatric, oral condition. 


\section{INTRODUCCIÓN}

La infancia, la adolescencia, la adultez y la vejez son etapas del desarrollo humano ${ }^{1}$. La vejez al igual que todas las etapas manifiesta una serie de cambios en diferentes niveles, pero hacer una distinción entre salud y enfermedad aún genera controversia, por la falta de claridad entre lo que se considera normal y anormal para este grupo de edad. ${ }^{2}$

La sociedad envejece rápidamente como efecto del aumento en la expectativa de vida y el descenso en la nata$\operatorname{lidad}^{2,3}$. En algunos países las personas de más de 60 años representan entre un 15 y $16 \%$ de la población total ${ }^{4}$. Este fenómeno tiene implicaciones de todo tipo por la importancia que tiene lograr salud y bienestar para todos sin excluir este grupo en particular. ${ }^{5}$

Los cambios asociados con la vejez, son también evidentes a nivel del sistema estomatognático $0^{2,3} \mathrm{y}$ producto de las modificaciones normales que suceden a nivel de cada una de sus estructuras; pero también como efecto de la condición sistémica, factores sociales, mentales y ambientales, que afectan al individuo. ${ }^{2}$

Algunos estudios realizados muestran que la incidencia de caries dental es mayor en personas de la tercera edad que en la población infantil ${ }^{6}$. El III Estudio Nacional de Salud Bucal, realizado en Colombia, en 1998, mostró que la mortalidad dentaria ha pasado a ser un problema fundamental de la población adulta y que, a diferencia de lo observado en el estudio anterior, la frecuencia es muy baja en la población joven.?

La odontogeriatría es un área naciente en el mundo entero. En nuestro país sólo la Universidad Nacional de Colombia presenta oficialmente en su programa académico un semestre dedicado exclusivamente a la atención del adulto mayor. ${ }^{8}$

En Bucaramanga no se conocen investigaciones que ilustren las necesidades orales de nuestras personas ancianas, menos aún de los adultos mayores institucionalizados. Se acepta, que la implementación de programas de salud oral dirigidos a grupos particulares debe fundamentarse en las necesidades detectadas. Por lo anterior, el propósito de este estudio es establecer el perfil orofacial de las personas adultas mayores institucionalizadas de la ciudad de Bucaramanga y su área metropolitana, con el fin de orientar, en el futuro, programas dirigidos a la aten- ción, promoción de la salud y prevención de la patologías bucodentales, que respondan a las necesidades detectadas.

\section{MATERIALES Y MÉTODOS}

Se realizó un estudio observacional descriptivo $0^{9}$ con el fin de determinar la prevalencia de las condiciones orales del grupo objeto de investigación. La población en estudio estuvo representada por 1.117 adultos mayores, residentes permanentes de instituciones geriátricas, en el municipio de Bucaramanga y su área metropolitana.

La muestra fue seleccionada mediante un muestreo aleatorio simple, con base en una prevalencia esperada de $45 \%$ de alteraciones dentofaciales, un margen de error de $5 \%$ y un $95 \%$ de confianza.

Se excluyeron del estudio individuos sordos o con retraso mental, incapacidad motriz severa, antecedentes de esquizofrenia o afasia, de acuerdo con su historia clínica, debido a la dificultad de obtener la información y realizar el examen oral.

Se estudiaron variables sociodemográficas como edad, género, afiliación a la seguridad social y condición laboral. El estado de salud general se determinó con base en los parámetros establecidos por el Ministerio de Salud en el estudio de morbilidad oral. ${ }^{\text {? }}$

El examen facial se categorizó en 8 zonas: cuero cabelludo, frente y sienes, región periorbitaria, mejillas, región perinasal, peribucal, submandibular y cuello observando la presencia de masas, manchas o úlceras. El examen de la articulación témporomandibular (ATM) consideró el índice de Helkimo y la presencia de ruido y dolor articular, palpación lateral, dolor en los músculos temporal, masetero, pterigoideo interno y externo, esternocleidomastoideo, trapecio; y, finalmente, apertura de boca.

Para la valoración de la mucosa se observó el surco vestibular, paladar, orofaringe, lengua, piso de la boca; se tuvieron en cuenta las alteraciones del color y la forma 0 la textura en cada una de estas estructuras. La determinación del estado de la dentición incluyó el COP(d) y el Índice de Caries Radicular (RCI), de acuerdo con los criterios establecidos por Katz, en la publicación de Wallace. ${ }^{10}$ 
La condición periodontal se estableció mediante el Índice Periodontal Comunitario y las necesidades de tratamiento (CPITN), según lo establecido en el ENSAB III. ${ }^{7}$

La placa dentobacteriana y el nivel de higiene oral se determinaron según el índice cuantificado de placa (ICP). ${ }^{11}$ El estado protésico se estableció con base en el estudio de morbilidad oral (ENSAB III). ${ }^{7}$

Previa recolección de la información, los estudiantes fueron estandarizados en técnicas y procedimientos para registrar en forma óptima los hallazgos en la cavidad oral, con la participación de varios especialistas.

\section{Análisis}

Los datos se analizaron aplicando medidas de estadística descriptiva ${ }^{12}$, según la naturaleza y distribución de cada variable, en la totalidad de los individuos incluidos en el estudio. Se describieron las características sociodemográficas y clínicas, los antecedentes médicos, la evaluación orofacial, de la ATM, la mucosa oral, COP(d), la caries radicular, CPITN, el ICP y el estado protésico. La base de datos se elaboró en Excel ${ }^{13}$ y el análisis en el programa STATA $6.0 .^{14}$

Se siguieron las recomendaciones éticas establecidas en la Resolución 008430, de 1993, del Ministerio de Salud de Colombia; según el título II en su artículo 11, clasifica éste estudio de riesgo mínimo.

\section{RESULTADOS}

\section{Descriptivo general}

De los 300 individuos seleccionados se evaluaron 284; el $57.4 \%$ pertenecían a instituciones geriátricas del municipio de Bucaramanga, el 15\% de Floridablanca, el 10.3\% de Girón y el 17.3\% de Piedecuesta (Tabla 1).

La distribución por género mostró un $45 \%$ femenino y $55 \%$ masculino, con un promedio de $77.7 \pm 8.8$ años y un rango entre 60 y 96 años. El 11.7\% de la población está activa laboralmente, pues desempeñan algún oficio dentro de la institución. El 35\% de la población no tiene seguridad social; de los vinculados a algún régimen de seguridad social el 21\% pertenece al régimen contributivo, el $40 \%$ al subsidiado; el resto registró medicina prepagada.
En el análisis de la condición general, la mayor frecuencia fue para el consumo de medicamentos de control, $51.8 \%$, seguido por hipertensión arterial, 39\%; alteraciones del sistema nervioso, 38\% (Tabla 2).

La mayor prevalencia de alteraciones faciales se registró en la mejilla, en el 80.6\%, y de éstas las manchas fue la condición que se observó con mayor frecuencia, en $96 \%$. En segundo y tercer lugar de alteraciones aparecen la frente-sienes y región periorbitaria, cuya condición prevalente también fueron las manchas. Las masas predominaron en la región perinasal y periorbitaria. Las úlceras se observaron más en la frente y mejillas, con la misma frecuencia (Tabla 3).

El 61\% presentó algún tipo de sintomatología en la ATM. El músculo pterigoideo externo mostró mayor prevalencia de sintomatología dolorosa en el $44 \%$, seguido del músculo pterigoideo interno. El 8.8\% de la población registró, apertura de la boca disminuida (Tabla 4).

Las alteraciones en la mucosa oral reflejan una prevalencia elevada de lesiones en la lengua y bordes laterales (66.2\%), principalmente de color blanco. En segundo lugar se encuentran las lesiones en la mucosa bucal (61.3\%) con lesiones pigmentadas y rojas, y en tercer lugar en el labio con lesiones pimentadas y en el piso de la boca y la lengua ventral con lesiones rojas (Tabla 5).

Tabla 1. Instituciones geriátricas visitadas

\begin{tabular}{|c|c|c|c|}
\hline Instituciones & Número & Porcentaje & Cudad \\
\hline Asilo San Antonio & 52 & 17.33 & Bucaramanga \\
\hline Asilo San Rafael & 60 & 20.00 & Bucaramanga \\
\hline Bienestar del Anciano & 34 & 11.33 & Bucaramanga \\
\hline Ensèname a Enwejecer & 3 & 1.00 & Bucaramanga \\
\hline Hogar luz de Esperanza & 6 & 2.00 & Bucaramanga \\
\hline Hogar Viejtos Queridos & 9 & 3.00 & Bucaramanga \\
\hline Mi Dulce Hogar & 4 & 1.33 & Bucaramanga \\
\hline Sueftos de VWir & 2 & 0.67 & Bucaramanga \\
\hline Vida a la Vejez & 2 & 0.67 & Bucaramanga \\
\hline Bienestar del Anciano & 45 & 15,00 & Floridablanca \\
\hline Señor de los Milagros & 31 & 10.33 & Girón \\
\hline Asilo San Francisco Javier & 52 & 17.33 & Piedecuesta \\
\hline
\end{tabular}


El Índice Cuantificado de Placa no pudo evaluarse en el 65.14\% de la población, por ausencia de piezas dentales. En los que se pudo aplicar el control de placa, se estableció que el 9.1\% tenía un ICP bueno, el 20.2\% regular y el $70.7 \%$ malo.

Tabla 2. Antecedentes médicos registrados

\begin{tabular}{lcc}
\hline Antecedentes médicos & Número & Porcentaje \\
\hline Diabetes & 45 & $16 \%$ \\
Hipertensión Arterial HTA & 111 & 39 \\
Problemas renales & 62 & 22 \\
Incontinencia urinaria & 43 & 15.1 \\
Osteosporosis & 19 & \\
Nervios (Estrés) & 110 & 38.73 \\
Cardiovasculares & 62 & 21.83 \\
Enfermedades hepáticas & 8 & 2.83 \\
Tratamiento médico & 66 & 23.24 \\
Medicamentos de control & 147 & \\
N=284 & & \\
\hline
\end{tabular}

En cuanto al COP-D, se encontró un promedio de cariados de 3, rango (0-23), obturados 0.3 (0-9), perdidos 25 (0-28), para un total de COP-D en promedio de 27 con un rango de (8-28). Es importante destacar que el $73 \%$ de la población tenía un COP-D de 28.

El Indice de Caries Radicular se pudo establecer en 131 individuos, con un rango entre 0 y 50 , promedio de $31 \mathrm{y}$ mediana de 40. Al analizar las frecuencias se encontró que el $25 \%$ de la población presentaba un RCI de cero, el $7.63 \%$ entre 1 y $20,18.3 \%$ de 21 a 40 y el $32.8 \%$ de 41 a 50 .

El estado periodontal no pudo ser evaluado en 192 (67.6\%), por la misma razón que el COP-D, de los 92 restantes, el $23.9 \%$ mostraron bolsas mayores de $6 \mathrm{~mm}$. (periodontitis avanzada), el 39.1\% presentó bolsas de 4 a $5 \mathrm{~mm}$ con presencia de cálculo y placa subgingival (periodontitis moderada), en el $21.7 \%$ se detectó presencia de cálculos supragingivales (periodontitis incipiente), el 8.7\% demostró sangrado al sondaje (gingivitis marginal) y tan sólo el $6.5 \%$ presentó un periodonto sano.

Se encontró edentulismo total en el 55\% de la población. Al analizar el estado protésico el $34.1 \%$ y el $53.5 \%$ de la población no tiene prótesis en los maxilares superior e inferior respectivamente. Se destaca la frecuencia de necesidad de elaboración y cambio, $30 \%$ en el maxilar inferior y $44.7 \%$ en el superior, respectivamente (Tabla 6.)

No se detectaron asociaciones significativas entre la presencia de prótesis o necesidad de cambio con alteraciones en la mucosa oral.

Tabla 3. Valoración Facial

\begin{tabular}{lccccc}
\hline Examen facial & \multicolumn{2}{c}{ Anormal } & Masas & Manchas & \multicolumn{2}{c}{ Úlceras } \\
& $\mathrm{N}$ & $\%$ & $\mathrm{~N}$ & $\mathrm{~N}$ & $\mathbf{N}$ \\
\hline Cuero cabelludo & 110 & 38.7 & 19 & 104 & 8 \\
Frente y Sienes & 214 & 75.35 & 75 & 204 & 18 \\
Periorbitaria & 209 & 73.59 & 82 & 197 & 8 \\
Mejillas & 229 & 80.63 & 70 & 222 & 18 \\
Perinasal & 205 & 72.18 & 83 & 199 & 9 \\
Peribucal & 175 & 61.62 & 44 & 160 & 3 \\
Submandibular & 121 & 42.61 & 23 & 115 & 1 \\
Cuello & 158 & 55.63 & 64 & 140 & 1 \\
\hline N=284 & & & & & \\
\hline
\end{tabular}




\section{DISCUSIÓN}

El primer paso para la implementación de programas de salud oral en los adultos mayores es el diagnóstico de su condición oral, ya que no existe una caracterización precisa de la salud bucal de este grupo poblacional.,6

Este trabajo se propuso como objetivo, identificar el perfil orofacial del paciente adulto mayor institucionalizado, de Bucaramanga y su área metropolitana, como punto de partida para la implementación de programas asistenciales, de promoción de la salud oral y de prevención de las alteraciones bucodentales.
Los hallazgos muestran, con claridad, que la valoración de la condición oral no debe restringirse a la condición dental o periodontal, pues en el nivel del sistema estomatognático, son muchas las estructuras que cambian, como efecto del envejecimiento; es fundamental que el odontólogo sea consciente de este proceso y tenga habilidad para relacionar la salud oral con la condición sistémica del individuo. ${ }^{15}$

La condición general debe ser incluida en el análisis, pues existe una relación directa entre la pobre salud general y una pobre salud oral, debido a enfermedades concomitantes y un sistema inmune debilitado y sometido a diversas medicaciones. ${ }^{16}$

Tabla 4. Valoración de la ATM

\begin{tabular}{|c|c|c|c|c|}
\hline \multirow[b]{2}{*}{ ATM } & \multicolumn{2}{|c|}{ Anormal } & \multirow{2}{*}{$\begin{array}{c}\text { Unilateral } \\
\qquad \mathrm{N}\end{array}$} & \multirow{2}{*}{$\begin{array}{c}\text { Bilateral } \\
\text { N }\end{array}$} \\
\hline & N & $\%$ & & \\
\hline $\begin{array}{l}\text { Ruido articular } \\
\text { Dolor en: }\end{array}$ & 170 & 59.86 & 74 & 96 \\
\hline Palpación lateral & 86 & 30.28 & 27 & 59 \\
\hline Músculo temporal & 30 & 10.56 & 6 & 24 \\
\hline Músculo masetero & 73 & 25.7 & 21 & 52 \\
\hline Músculo Pterigoideo Int. & 86 & 30.28 & 42 & 44 \\
\hline Músculo Pterigoideo Ext. & 126 & 44.37 & 33 & 93 \\
\hline Músculo Esternocleidomastoideo & 67 & 23.6 & 25 & 42 \\
\hline Músculo Trapecio & 78 & 27.46 & 15 & 63 \\
\hline Apertura de la boca & 25 & 8.8 & & \\
\hline Apertura inferior a $40 \mathrm{~mm}$ & 103 & 36.3 & & \\
\hline
\end{tabular}

Tabla 5. Valoración de la Mucosa Oral

\begin{tabular}{|c|c|c|c|c|c|c|c|c|}
\hline \multirow{3}{*}{$\begin{array}{l}\text { Localización } \\
\text { de la lesión }\end{array}$} & \multicolumn{2}{|c|}{ Anormal } & \multicolumn{3}{|c|}{ Color } & \multicolumn{3}{|c|}{ Tipo de lesión } \\
\hline & $\mathrm{N}$ & $\%$ & Blanca & Roja & Pigmentada & $\begin{array}{l}\text { Vesiculo } \\
\text { ampollosa }\end{array}$ & Ucerosa & Exofitica \\
\hline & & & $\mathrm{N}$ & $\mathrm{N}$ & $\mathrm{N}$ & $\mathrm{N}$ & $\mathrm{N}$ & $\mathrm{N}$ \\
\hline Labios & 155 & 54.58 & 5 & 29 & 98 & 6 & 16 & 33 \\
\hline Mucosa bucal & 174 & 61.27 & 25 & 70 & 82 & 9 & 5 & 38 \\
\hline $\begin{array}{l}\text { Piso de boca y } \\
\text { lengua ventral }\end{array}$ & 155 & 54.58 & 10 & 98 & 46 & 4 & 4 & 17 \\
\hline $\begin{array}{l}\text { Lengua y bordes } \\
\text { laterales }\end{array}$ & 188 & 66.20 & 157 & 21 & 19 & 1 & 5 & 7 \\
\hline $\begin{array}{l}\text { Paladar duro } \\
\text { y blando }\end{array}$ & 144 & 50.7 & 10 & 101 & 18 & 1 & 4 & 10 \\
\hline Reborde alveolar & 151 & 53.17 & 9 & 105 & 16 & 2 & 11 & 52 \\
\hline
\end{tabular}

$\mathrm{N}=284$ 
A pesar de la afirmación de que la pérdida de dientes, en las personas ancianas ha disminuído, el presente estudio muestra edentulismo en el 92\% de la población, aspecto que no coincide con lo expresado por Baldoni et $\mathrm{al}^{2}$, cuando citan el estudio de Miyasaki con una prevalencia de edentulismo en el $27 \%$ de la población adulta mayor. Esto sugiere que los indicadores para población institucionalizada son nada alentadores.

Nuestros resultados no coinciden con las cifras presentadas en el ENSAB III en el que se afirma que el 51.9\% de las personas de 15 a 44, y 55 años, y más, mantienen la dentición completa en el maxilar superior y el 46.4 \% para el maxilar inferior. La evaluación, de grupos en edades diferentes al analizado en esta investigación, podría explicar esta diferencia, sumada a la condición de institucionalizada de esta población.

Baldoni et $a l^{2}$ y Munevar et $a l^{5}$ coinciden en que la higiene oral de los pacientes adultos mayores es deficiente, quizá debido a las limitaciones físicas, mentales y/o a los hábitos de autocuidado, que muestran los pacientes institucionalizados, que deben convertirse en un criterio por analizar, al proponer e implementar estrategias de prevención de la enfermedad y promoción de la salud.
No es posible comparar las alteraciones orofaciales de este estudio con otros trabajos, pues no se encontraron otras investigaciones que especifiquen con detalle este aspecto en adultos mayores; sin embargo, es fundamental reconocer que es compromiso del odontólogo no dejar de lado el riesgo que representan para el sistema estomatognático, las lesiones en las estructuras que lo componen, ya que se ha detectado la presencia de una lesión maligna por lo menos en el 32.5\% de los sujetos fallecidos, de más de 60 años de edad. ${ }^{2}$

Las alteraciones de la ATM se han registrado en el 30\% de los pacientes adultos mayores ${ }^{2}$. En el ENSAB III el ruido articular registra una prevalencia del $29.6 \%$ en mujeres y $21.7 \%$ de los hombres, aspecto considerado relevante al relacionarlo con el presente estudio, pues la frecuencia de sintomatología asociada a la ATM y a los ruidos articulares fue cercana al $60 \%$ de nuestra población. Quizás esto se explique por el índice que se empleó en el primer estudio, además, del grupo diferente de edad evaluado.

Los resultados de este trabajo permiten concluir que el perfil orofacial es el primer paso, y muy importante, para implementar procesos integrales, orientados a preservar la salud oral en este grupo de edad. Es necesario, además,

Tabla 6. Valoración del estado protésico

\begin{tabular}{|c|c|c|c|c|}
\hline \multirow[b]{2}{*}{ Presencia de prótesis } & \multicolumn{2}{|c|}{ Maxilar Superior } & \multicolumn{2}{|c|}{ Maxilar Inferior } \\
\hline & $\mathrm{N}$ & $\%$ & $\mathbf{N}$ & $\%$ \\
\hline $\operatorname{Sin}$ & 97 & 34.1 & 152 & 53.5 \\
\hline Prótesis fija & 0 & 0 & 0 & 0 \\
\hline Prótesis removible & 12 & 4.2 & 21 & 7.39 \\
\hline Prótesis fija y removible & 1 & 0.35 & 0 & 0 \\
\hline Prótesis total & 173 & 60.9 & 110 & 38.7 \\
\hline No aplica & 1 & 0.35 & 1 & 0.35 \\
\hline Necesidad de cambio & 127 & 44.7 & 79 & 27.8 \\
\hline \multicolumn{5}{|l|}{ Necesidad de elaboración: } \\
\hline Prótesis parcial & 38 & 13.4 & 83 & 29.2 \\
\hline Prótesis total & 61 & 21.5 & 72 & 25.4 \\
\hline
\end{tabular}


practicar una valoración integral de la persona mayor, con el fin de identificar otros posibles factores, que pueden alterar su condición oral.

Es necesario, también, enfatizar que la condición oral del paciente adulto mayor institucionalizado, en nuestra región, es deficiente y que deben hacerse grandes esfuerzos para modificar positivamente este perfil.

\section{BIBLIOGRAFÍA}

1. Prieto 0, Vega E. Temas de gerontología. Ed. Científico Técnica. La Habana. 1996, p. 188

2. Baldóni M, Balucchi L, Campo J. El envejecimiento de la cavidad oral y de sus tejidos. Fundamentos en Odontología. Antonio Bascones. Sección XXVIII. Odontogeriatría. Ediciones Médico-Dentales, S.L. Segunda Edición, Madrid 1998 p.3489, 3495

3. Ast F, Bjorn H. Odontología Geriátrica. Labor do Brasil, Rio de Janeiro. 1973

4. Hattori Y, Kikuchi M, Tamazawa Y, Watanabe M. Dental treatment of homebound patients in Sendai. Dentistry in Japan. 2001; 37:155-157.

5. Munevar AM, Rojas JK, Marín D. Perfil epidemiológico bucal de pacientes de 55 años y más que asisten a las clínicas del geronte a la facultad de odontología de la U. N. durante el primer semestre del año 2000 y cuál es la percepción que tienen estos pacientes sobre su propio estado bucal. Federación Odontológica Colombiana. 2002; 61:732.

6. Irigoyen ME, Velásquez C, Zepeda MA, Mejía A. Caries dental y enfermedad periodontal en un grupo de personas de 60 años o más de la ciudad de México. Revista de la Asociación dental Mexicana 1999; 56:64-69

7. Ministerio de Salud. Estudio Nacional de Salud Bucal-ENSAB III. Bogotá, Colombia. Tomo VIII, 1999. p. 109-99.

8. Marín DJ. Es necesaria la Odontogeriatría?. Revista de la Federación Odontológica Colombiana. Salud para la tercera edad 2000; 59:39-44.

9. Rothman KJ, Greenland S. Modern Epidemiology. Second Ed. Lippincott-Raven Publishers, East Washington Square, Philadelphia, PA, 1998.

10. Wallace MC, Retief DH, Bradley EL. Prevalence of root caries in a population of older adults. Gerodontics 1988; 4:84-89.

11. Pieschacón MP. Programa piloto de odontología preventiva con escolares del municipio de Bucaramanga. Bucaramanga, Colombia: (1998). Tesis de grado.

12. Pagano M, Gauvreau K. Principles of biostatistics. Duxbury press, Belmont, 1993.
13. Microsoft Excell, 5.0, Microsoft Corporation, 1997

14. StataCorp. 1999. Stata Statistical Software: Release 6.0. College Station, TX: Stata Corporation.

15. Axelson P. An introduction to risk prediction and preventive dentistry. Quintessense Publishing, Illinois, USA, 1999.

16. Pajukoski H, Meurman JH, Snellman-Grohn S, Sulkava R. Salud oral en pacientes ancianos hospitalizados y no hospitalizados habitantes de una comunidad. Oral Surg Oral Med Oral Pathol Oral Radiol Endod 1999; 88:437-43. 